\title{
Padrões alimentares de adolescentes obesos e diferentes repercussões metabólicas ${ }^{1}$
}

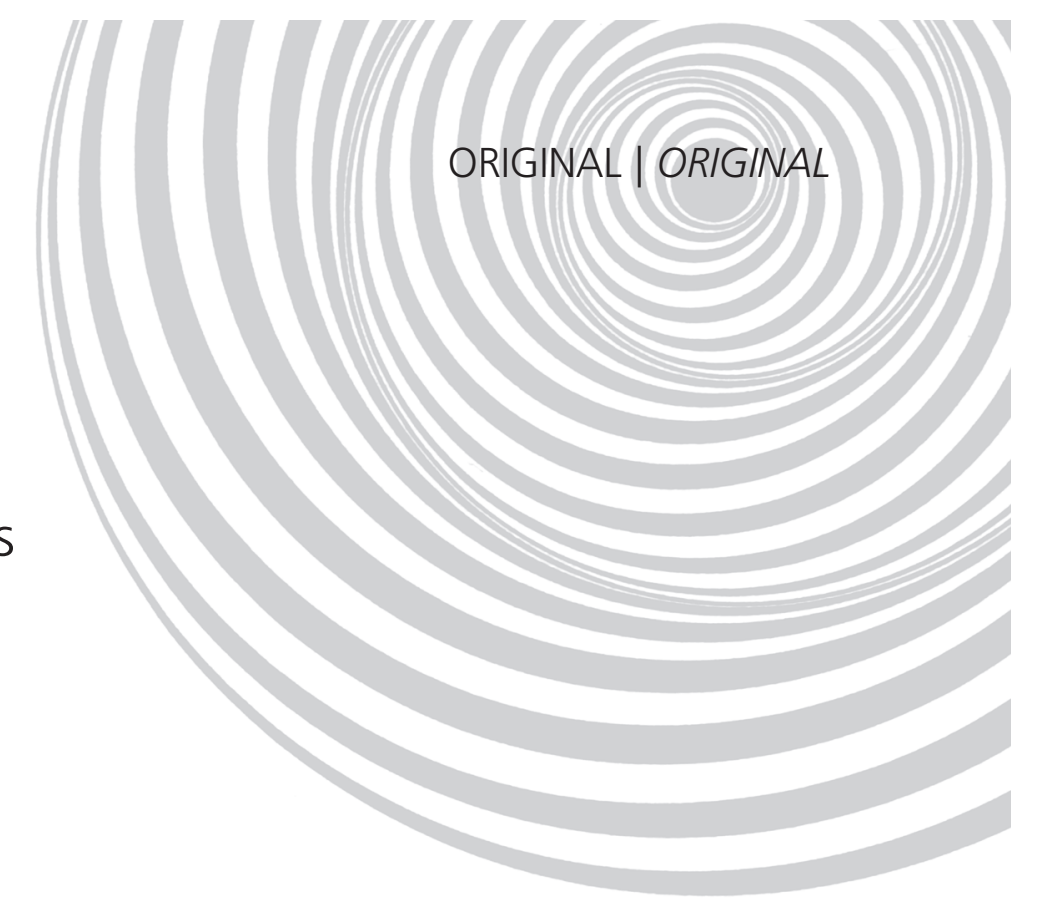

\section{Dietary patterns of obese adolescents and different metabolic effects}

Vera Regina Mello DISHCHEKENIAN²

Maria Arlete Meil Schimith ESCRIVÃO2

Domingos PALMA ${ }^{2}$

Fábio ANCONA-LOPEZ²

Eutália Aparecida Candido de ARAÚJO3

José Augusto de Aguiar Carrazedo TADDEI ${ }^{2}$

\section{RE S U M O}

\section{Objetivo}

Avaliar a associação de padrões alimentares com alterações metabólicas em adolescentes obesos.

\section{Métodos}

Estudo transversal envolvendo 76 estudantes (ambos os sexos, 14-19 anos, IMC $\geq$ P95 e Tanner $\geq 4$ ) de escolas públicas da cidade de São Paulo, Brasil. Foram coletados dados antropométricos e bioquímicos, e aplicados questionários com informações sociodemográficas e registro alimentar de 4 dias, entre 2006 e 2007. Escores fatoriais foram obtidos na análise fatorial e, após ajustes, três padrões alimentares identificados foram associados a fatores de risco biológico por regressão linear múltipla.

\section{Resultados}

O padrão Tradicional (arroz e massas, feijões, carnes vermelhas, embutidos, óleos e doces) foi positivamente associado com insulina, glicemia e triglicérides e negativamente associado com lipoproteína de alta densidade. O padrão Em Transição (peixe, aves, ovos, pães, manteiga, leite e derivados, hortaliças, frutas, sucos de frutas e açúcar refinado) apresentou as mesmas associações, além de associação similar com a pressão arterial diastólica. O padrão Fast Food (cafeteria, hambúrguer, maionese, bolacha, bolos e tortas, chocolate e refrigerantes) apresentou associação positiva com o colesterol, lipoproteína de baixa densidade e pressão arterial sistólica e diastólica, e associação negativa com insulina e lipoproteína de alta densidade.

1 Artigo elaborado a partir do projeto FAPESP, intitulado "Fatores de risco e comorbidades associados à obesidade em adolescentes de escolas públicas da cidade de São Paulo", com registro nº 0754490-8R.

2 Universidade Federal de São Paulo, Escola Paulista de Medicina, Departamento de Pediatria. R. Loefgreen, 1647, 04040-032, São Paulo, SP, Brasil. Correspondência para/Correspondence to: J.A.A.C. TADDEI. E-mails: <taddei.dped@epm.br>; <nutsec@yahoo.com.br>.

${ }^{3}$ Universidade de São Paulo, Escola de Enfermagem, Departamento Médico-Cirúrgico. São Paulo, SP, Brasil. 
18 | V.R.M. DISHCHEKENIAN et al.

\section{Conclusão}

Os padrões Tradicional e Em transição apresentaram associações diferentes com o metabolismo lipídico e glicídico quando comparados com o padrão Fast Food. Os três padrões podem ser considerados obesogênicos, porém o padrão Fast Food pareceu ser o mais aterogênico e promotor de hipertensão arterial.

Termos de indexação: Adolescente. Comportamento alimentar. Consumo alimentar. Doenças cardiovasculares. Fatores de risco. Obesidade.

\section{A B S T R A C T}

\section{Objective}

This study assessed the association between dietary patterns and metabolic changes in obese adolescents.

\section{Methods}

This was a cross-sectional study of 76 students (both genders, 14-19 years old, BMI $\geq P 95$ and Tanner stage $\geq 4$ ) enrolled in public schools from 2006 to 2007 in São Paulo city, Brazil. A sociodemographic questionnaire and 4-day food record were administered and anthropometric and biochemical data were collected. Scores for dietary patterns were assessed by factor analysis and after potential confounders were controlled, multilinear regression was used to associate the three identified patterns with biological risk factors.

\section{Results}

The Traditional Pattern (rice and cereals, beans, red meat, sausage, oils and sweets) was positively associated with insulin, blood glucose and triglycerides and negatively associated with high-density lipoprotein. The In-Transition pattern (fish, poultry, eggs, bread, butter, milk and dairy products, vegetables, fruits, fruit juice and white sugar), presented the same positive associations in addition to an association with diastolic blood pressure. The Fast Food pattern (high fat bakery products, hamburger, mayonnaise, cookies and crackers, chocolate and sodas) presented a positive association with cholesterol, low-density lipoprotein, systolic and diastolic blood pressure and a negative association with insulin and high-density lipoprotein.

\section{Conclusion}

The Traditional and In-Transition patterns were differently related to glucose and lipid metabolism when compared with the fast food pattern. The three patterns could be considered obesogenic, however the Fast Food pattern seems to be the most atherogenic and promoter of hypertension.

Indexing terms: Adolescent. Feeding behavior. Food consumption. Cardiovascular diseases. Risk factor. Obesity.

\section{N T R O D U ÇÃ O}

A obesidade em adolescentes é um problema crescente mundialmente. Suas implicações na saúde poderão repercutir nas taxas de diabetes e doenças cardiovasculares alguns anos mais tarde ${ }^{1}$. Diversos estudos epidemiológicos têm acompanhado a transição nutricional, com o objetivo de identificar fatores associados a alterações nutricionais e suas repercussões metabólicas ${ }^{2-5}$. A obesidade normalmente encontra-se associada à resistência insulínica e à hiperinsulinemia, consideradas mediadoras de alterações como dislipidemias, doença cardiovascular, diabetes tipo 2, hipertensão e doenças crônicas. Considerando-se que a instalação da obesidade dá-se desde a infância, é de fundamental importância seu monitoramento desde então, até a adolescência e idade adulta jovem ${ }^{6}$.

No Brasil, os resultados da Pesquisa de Orçamentos Familiares (POF) revelam aumento do excesso de peso entre crianças e adolescentes, atingindo aproximadamente $15 \%$ das meninas e $18 \%$ dos meninos entre 10 e 19 anos $^{7}$.

A obesidade pode ter início ou ser agravada na adolescência, devido ao aumento fisiológico do tecido adiposo, manifestado especialmente no sexo feminino ${ }^{8}$. Muitos fatores parecem interferir no consumo alimentar nessa fase, tais como valores socioculturais, imagem corporal, grupo social, renda familiar, alimentos consumidos 
fora de casa, aumento do consumo de alimentos altamente energéticos, influência exercida pela mídia e grupo de convívio, omissão de refeições, distância entre a casa, escola e trabalho, disponibilidade e facilidade de preparo dos alimentos, além de instabilidade emocional.

Na sociedade moderna, o crescimento da indústria alimentícia associado à maior acessibilidade aos alimentos, aumento da participação da mulher no mercado de trabalho, ampliação das redes de comercialização de alimentos (fast food, cantinas escolares, lanchonetes, quiosques e restaurantes) está ditando modos de consumo alimentar e consequente entrada de novos produtos alimentares em substituição aos alimentos tradicionalmente consumidos ${ }^{9}$.

A importância da dieta na etiologia das doenças tem sido evidenciada pelo crescente número de estudos epidemiológicos, uma vez que o consumo alimentar habitual constitui um dos principais fatores determinantes passíveis de modificações para as Doenças Crônicas Não-Transmissíveis (DCNT) ${ }^{10-12}$. Muitas vezes, doenças não surgem da escassez de nutrientes, mas da forma combinada como eles interagem. Procura-se cada vez mais descobrir padrões alimentares que predisponham ao ganho de gordura corporal, a fim de implantar políticas de saúde pública que combatam o problema ${ }^{6,13}$.

Paralelamente, há um crescente interesse em se usar índices de qualidade de dieta para avaliar se um determinado padrão diminui o risco de certa doença. Um exemplo disso é a Dieta Mediterrânea, caracterizada pela riqueza em cereais integrais, frutas, vegetais e elevada proporção de gorduras monoinsaturadas em relação às saturadas $^{14}$.

O conceito de que o consumo de alimentos em conjunto pode ser mais importante do que os nutrientes analisados individualmente não é recente. Em 1982, Schwerin et al. ${ }^{2}$ aplicaram pela primeira vez métodos estatísticos para converter o consumo alimentar em algo mais significativo do que componentes isolados da dieta. Surgiram a partir daí as primeiras associações de padrões alimentares com fatores de risco à saúde e em 1998 foi publicado o primeiro estudo, que associou padrões alimentares, definidos por análise fatorial, com câncer de cólon².

Considerando os resultados desse estudo, surgiu a hipótese de que os padrões de consumo alimentar poderiam apontar associações entre alimentação e fatores de risco, de forma melhor que qualquer alimento ou nutriente isolado. A confirmação dessa tese pode ser traduzida pelo crescente número de trabalhos utilizando padrões alimentares como fator prognóstico na relação entre alimentação e risco de doenças crônicas, especialmente quando estas estão associadas a várias características da dieta. Tal tendência vai ao encontro da atual preconização da World Health Organization (WHO) ${ }^{1}$, que sugere que as recomendações alimentares para populações sejam baseadas em alimentos e não em nutrientes. As associações encontradas devem ser simples, facilmente interpretadas e passíveis de se converter em conhecimentos que possam ser transformados em condutas aplicáveis em nível de saúde pública ${ }^{15}$. Ainda existem poucos estudos com adolescentes ${ }^{3,16-18}$ e nenhum anterior brasileiro definindo padrões alimentares de adolescentes obesos.

Diante da complexidade de fatores relacionados ao hábito alimentar do adolescente e sua repercussão na saúde na vida adulta, o presente estudo tem por objetivo identificar o perfil de consumo alimentar de adolescentes obesos e sua associação com doenças crônicas, uma vez que os fatores dietéticos, por meio de sua exposição disseminada, exercem importante impacto sobre as condições de nutrição e saúde da população.

\section{MÉ TOD OS}

Trata-se de estudo de delineamento transversal com adolescentes de 14 a 19 anos de ambos os sexos, classificados como obesos por Índice de Massa Corporal (IMC) $\geq$ P95 pelo critério de Must et al. ${ }^{19}$, em estadiamento puberal $\geq 4$ pelo 
critério de Tanner ${ }^{20}$, e selecionados em quatro escolas públicas de ensino médio na região da Vila Mariana, na cidade de São Paulo (SP), Brasil, entre agosto de 2006 e maio de 2007. Dos 150 alunos obesos identificados, 124 (82,66\%) preencheram os critérios de seleção, sendo que 42 $(33,87 \%)$ se recusaram a participar, $2(1,61 \%)$ mudaram de escola durante o processo seletivo e $4(3,22 \%)$ não trouxeram o registro alimentar preenchido, o que resultou na amostra de 76 adolescentes que completaram todas as etapas da pesquisa. Não foram incluídos na amostra adolescentes que apresentaram doenças agudas, doenças crônicas que não a obesidade, nem gestantes. Para evitar eventual viés, foi feita a comparação da distribuição de IMC, por sexo e idade, entre os participantes e os indivíduos que se recusaram a participar, não tendo sido encontradas diferenças significantes.

A coleta de dados incluiu antropometria, registro alimentar de 4 dias e questionário pré-codificado e pré-testado, aplicado por pediatras e nutricionistas da equipe, contendo informações sobre antecedentes pessoais, familiares, nível socioeconômico, dados comportamentais, alimentares e de atividade física.

\section{Avaliação clínica}

As medidas antropométricas de peso e estatura foram aferidas na primeira consulta, pelas nutricionistas da equipe, no ambulatório de Obesidade da Universidade Federal de São Paulo (UNIFESP). O peso foi avaliado por balança tipo plataforma digital, da marca Kratos ${ }^{\circledR}$, modelo "Linea", com capacidade mínima de 1,25kg e máxima de $150 \mathrm{~kg}$, e precisão de 50 gramas. A estatura foi medida com o estadiômetro portátil Alturexata ${ }^{\circledR}$, com escala em milímetros. As medidas foram realizadas duas vezes, obtendo-se a média como valor final. A Pressão Arterial Sistólica (PAS) e Pressão Arterial Diastólica (PAD) foi aferida pela médica do projeto, após período não inferior a 10 minutos em repouso sentado, utilizando-se esfigmomanômetro de mercúrio (marca Thycos ${ }^{\circledR}$ ), com manguito adequado para cada indivíduo, sendo considerado o valor médio de três aferições com intervalos de 5 minutos.

\section{Avaliação bioquímica}

Os alunos foram submetidos a coleta de sangue por meio de punção venosa, após 12 horas de jejum, para a determinação dos níveis de colesterol sérico total e frações, triglicérides, glicemia e insulina de jejum. Segue a descrição dos exames bioquímicos utilizados no presente estudo:

a) Glicemia de jejum: foi dosada pelo método enzimático, utilizando enzima hexoquinase e glicose-6-fosfato desidrogenase (Advia Chemistry System 1650 - Bayer);

b) Insulina de jejum: dosada pelo método imunoenzimático (Tosoh-Tosoh Corporation, Tokyo, Japan);

c) Lipoproteína de alta densidade-coles terol (HDLc) e Triglicérides (TG): dosados por método de Espectrofotometria de refletância - colorimétrico - 540 nm (Vitros Systems Chemistry 750 XRC - Ortho-Clinical Diagnostics, Inc., Johnson \& Johnson Company, New York, USA).

d) Lipoproteína de baixa densidade-colesterol (LDLC): obtido da equação de Friedewald: $L D L C=(C T-H D L C+T G / 5)$.

\section{Avaliação de consumo alimentar}

Durante a primeira consulta, o nutricionista aplicou o recordatório de 24 horas e ofereceu informações detalhadas de preenchimento, para aumentar a precisão do registro alimentar de 4 dias (3 dias da semana e 1 dia do final de semana).

Foram inseridos alimentos que não apresentavam todos os seus macro e micronutrientes cadastrados na tabela de composição centesimal do software e que eram necessários a esta pesquisa, utilizando-se tabelas de composição centesimal que contemplavam tais nutrientes. Parale- 
lamente, foram atualizados todos os alimentos industrializados, a fim de verificar alterações na composição e no tamanho das embalagens e porções. Para avaliar a adequação quantitativa da ingestão habitual, foram utilizadas as recomendações das Recommended Dietary Allowance $(\text { RDA })^{21}$, que visam atender as necessidades nutricionais da população considerando idade, sexo e estado fisiológico, por meio do NutWin (Nutwin - Programa de Apoio à Nutrição, versão 1.5, Unifesp/EPM, São Paulo, 2002). Cabe ressaltar que as RDA foram utilizadas com o intuito de possibilitar comparações com os dados da primeira fase deste estudo, baseados naquelas recomendações. A avaliação do consumo energético e distribuição dos macronutrientes foi obtida por meio de conversão do consumo alimentar apontado no registro alimentar.

\section{Análise estatística}

Os dados foram avaliados quanto a sua consistência interna pelos pesquisadores, antes de serem liberados para digitação e transcritos em bancos de dados com dupla digitação seguida de validação, empregando-se o pacote estatístico Epi Info, versão 6.0 (Centers for Disease Control and Prevention, Atlanta, GA, USA, 1994).

O programa Statistical Package for the Social Science (SPSS, versão 13.0, Chicago, IL, USA, 1999) foi utilizado na análise fatorial por componentes principais, para definir os padrões alimentares, e na regressão linear múltipla, para associar os padrões alimentares com as alterações metabólicas relacionadas à obesidade detectadas por exames clínicos e bioquímicos.

\section{Análise fatorial}

Para identificar os padrões alimentares, empregou-se análise fatorial aos dados colhidos no registro alimentar, considerando-se a média da quantidade consumida nos quatro dias de cada alimento/preparação. Primeiramente constituíram-se 20 alimentos ou grupos de alimentos, a partir da semelhança em sua composição nutri- cional ${ }^{3,12}$. A ingestão alimentar foi ajustada pelo consumo total de energia, por meio do Método Residual ${ }^{10}$.

A primeira fase da análise de componentes principais verificou as seguintes suposições básicas do modelo: variáveis métricas, tamanho da amostra maior que $50^{22}$, matriz de correlação de variáveis com 22 correlações $\geq 0,30$.

A adequação do tamanho amostral à análise fatorial foi confirmada pelos valores do teste Kaiser-Meyer-Oblin (KMO) de 0,696 e pela significância do teste de esfericidade de Bartlett, menor que 0,001 . Verificou-se ainda a adequação de cada variável à análise fatorial, por meio da matriz de correlação anti-imagem, com valores maiores que 0,50 .

Num segundo momento, foi aplicada a análise fatorial por componentes principais aos 20 alimentos/grupos de alimentos, selecionando-se a rotação Varimax para obtenção de fatores não correlacionados entre si.

O número de fatores a serem extraídos na análise foi definido pelo Método de Análise Paralela, visando obter um número de fatores extraídos que possibilitasse a identificação de padrões interpretáveis ${ }^{23}$.

Por último, foram calculados os escores fatoriais dos padrões alimentares. Eles posicionam cada indivíduo segundo o grau de participação em cada padrão alimentar, possibilitando que cada um tenha um escore fatorial em todos os padrões identificados.

Alimentos com carga fatorial (correlação do alimento com o fator) $\leq 0,300$ em um componente foram considerados de baixa correlação, tendo sido eliminados: leite fermentado e semidesnatado, barra de cereal, frutas secas, refrigerantes diet/light, creme de leite e sanduíche natural.

\section{Regressão linear múltipla}

A associação entre os escores fatoriais e as variáveis clínicas e laboratoriais foi analisada de três maneiras: sem ajustes; com modelagem 
multivariada controlada por sexo, idade e cor; e com modelagem multivariada controlada por renda, escolaridade da mãe e IMC. Idade, IMC, escores fatoriais, renda familiar, escolaridade da mãe e variáveis clínicas e bioquímicas foram tratadas como variáveis contínuas e normalizadas. Foram categorizadas as variáveis sexo (masculino e feminino) e cor (branca ou não branca). O nível de significância adotado nas análises foi de $5 \%$.

O estudo foi aprovado pelo Comitê de Ética em Pesquisa da Universidade Federal de São Paulo, protocolo $n^{\circ} 2109$, tendo todos os participantes assinado o Termo de Consentimento Livre e Esclarecido antes de sua inclusão na amostra.

\section{RES U L T A D O S}

A amostra do estudo foi composta por 76 adolescentes, sendo 44 do sexo masculino e 41 de cor branca. Sua caracterização encontra-se apresentada na Tabela 1. Os meninos apresentaram médias de peso, altura e glicemia significantemente maiores que as meninas, resultado semeIhante aos de outros estudos ${ }^{11,24}$.

Foram definidos três padrões alimentares representando o consumo alimentar da população em estudo (Tabela 2). A denominação dos padrões procurou utilizar nomenclatura de outros trabalhos com métodos estatísticos similares.

O padrão denominado "Tradicional" representou 13,96\% da variância total, sendo composto predominantemente por arroz, massas, feijões, óleos, carnes vermelhas, embutidos e doces. O padrão denominado "Em Transição" incorporou 11,20\% da variância, caracterizando-se pelo consumo relevante de leite e derivados, pães, manteiga e margarina, carnes brancas, ovos,

Tabela 1. Caracterização da amostra de adolescentes obesos matriculados em escolas públicas da cidade de São Paulo (SP), 2006.

\begin{tabular}{|c|c|c|c|c|c|}
\hline & \multicolumn{2}{|c|}{ Meninos $(n=32)$} & \multicolumn{2}{|c|}{ Meninas $(n=44)$} & \multirow{2}{*}{$p^{*}$} \\
\hline & Média & Desvio-padrão & Média & Desvio-padrão & \\
\hline Idade (anos) & 15,90 & 1,09 & 16,00 & 1,20 & 0,9077 \\
\hline Peso (kg) & 97,49 & 13,57 & 89,36 & 14,13 & 0,0139 \\
\hline Altura (m) & 1,74 & 0,67 & 1,64 & 0,64 & 0,0000 \\
\hline IMC $\left(\mathrm{kg} / \mathrm{m}^{2}\right)$ & 32,20 & 4,14 & 33,01 & 4,48 & 0,4275 \\
\hline Glicemia (mg/dL) & 95,00 & 8,00 & 92,00 & 7,00 & 0,0287 \\
\hline Insulina $(\mu \mathrm{U} / \mathrm{mL})$ & 15,29 & 11,87 & 14,51 & 6,24 & 0,5253 \\
\hline Colesterol (mg/dL) & 149,00 & 31,85 & 155,00 & 31,50 & 0,3897 \\
\hline $\mathrm{TG}(\mathrm{mg} / \mathrm{dL})$ & 88,00 & 41,00 & 85,00 & 42,00 & 0,8056 \\
\hline $\mathrm{HDL}-\mathrm{c}(\mathrm{mg} / \mathrm{dL})$ & 46,00 & 8,00 & 49,00 & 10,00 & 0,1483 \\
\hline $\mathrm{LDL}-\mathrm{c} \quad(\mathrm{mg} / \mathrm{dL})$ & 85,00 & 26,00 & 89,00 & 24,00 & 0.6516 \\
\hline PAS (mmHg) & 118,00 & 10,00 & 114,00 & 11,00 & 0,0973 \\
\hline $\mathrm{PAD}(\mathrm{mmHg})$ & 71,00 & 9,00 & 70,00 & 9,00 & 0,4787 \\
\hline Renda familiar (S.M.) & 1,80 & 1,20 & 1,60 & 0,80 & 0,2312 \\
\hline Membros família (n) & 3,70 & 1,40 & 3,80 & 1,20 & 0,7178 \\
\hline Escolaridade materna ${ }^{\star \star}$ & 3,03 & 1,55 & 3,02 & 1,37 & 0.9799 \\
\hline VET (kcal) & 2064,00 & 595,00 & 1833,00 & 650,00 & 0,1181 \\
\hline Carboidrato (g) & 262,00 & 78,00 & 253,00 & 100,00 & 0,6772 \\
\hline Carboidrato (\%VET) & 51,22 & 7,05 & 53,73 & 6,09 & 0,1014 \\
\hline Proteína (g) & 77,00 & 22,00 & 67,00 & 23,00 & 0,0731 \\
\hline Proteína (\%VET) & 15,51 & 2,86 & 14,50 & 3,79 & 0,2104 \\
\hline Lípides (g) & 78,00 & 4,95 & 65,00 & 3,61 & 0,0373 \\
\hline Lípides (\%VET) & 33,27 & 1,16 & 31,77 & 0,71 & 0,2504 \\
\hline
\end{tabular}

* Valor de p<0,05; S.M.: salário-mínimo; VET: valor energético total diário; n: número.

* * Escolaridade materna: 1) fundamental incompleto; 2) fundamental completo; 3) médio incompleto; 4) médio completo; 5) superior incompleto e 6) superior completo. 
hortaliças e frutas, sucos e açúcar. Por último, o padrão denominado "Fast Food" correspondeu a 9,58\% da variância, sendo marcadamente composto por produtos de cafeteria, hambúrguer, maionese, bolachas, bolos, tortas, refrigerantes e chocolate. Os três padrões, em conjunto, explicaram $34,8 \%$ da variabilidade total dos dados, o que está dentro dos valores observados na literatura de epidemiologia nutricional, tendo em vista que esta aponta variância total entre $21,7 \%{ }^{3}$ e $29,2 \%{ }^{25}$ quando identificados até quatro padrões.

\section{Padrões alimentares e alterações bioquímicas}

As associações entre os escores fatoriais dos padrões alimentares e as variáveis clínicas e bioquímicas são representadas na Tabela 3.

Verificou-se que, nas três modelagens, o padrão Tradicional teve associação significante e positiva com insulina, glicemia e TG, e associação significantemente negativa com o HDLc, tanto sem ajuste, como quando ajustado por renda,

Tabela 2. Distribuição da carga fatorial dos padrões de consumo alimentar de adolescentes matriculados em escolas públicas da cidade de São Paulo (SP), 2006.

\begin{tabular}{|c|c|c|c|}
\hline \multirow{2}{*}{ Alimento ou grupo } & \multicolumn{3}{|c|}{ Padrões } \\
\hline & Tradicional & Transição & Fast Food \\
\hline $\begin{array}{l}\text { 1) Arroz e massas: arroz polido, batata, mandioca, inhame, batata doce, farofa, cuscuz, } \\
\text { farinha de milho, cereal matinal, macarrão, lasanha, nhoque, panqueca, sopas }\end{array}$ & 0,774 & 0,074 & 0,062 \\
\hline 2) Óleos: óleos e azeites & 0,763 & 0,069 & 0,063 \\
\hline 3) Feijões: feijão, ervilha, lentilha, grão-de-bico, soja & 0,737 & 0,290 & 0,010 \\
\hline 5) Embutidos: salsicha, linguiça, presunto, mortadela, peito de peru, salame, rosbife & 0,442 & 0,049 & 0,023 \\
\hline $\begin{array}{l}\text { 6) Doces: balas, chicletes, geleia, mel, pudins, gelatina, doces de frutas, leite condensado, } \\
\text { doces caseiros, doces industrializados e diet }\end{array}$ & 0,368 & 0,080 & 0,121 \\
\hline 7) Carnes brancas: aves e pescados & 0,186 & 0,654 & 0,092 \\
\hline 10) Hortaliças e frutas: verduras, legumes, frutas e molho de tomate & 0,031 & 0,519 & 0,281 \\
\hline 11) Ovos & 0,020 & 0,511 & 0,086 \\
\hline 12) Pães: pão francês, integral, de forma, torradas & 0,126 & 0,489 & 0,133 \\
\hline $\begin{array}{l}\text { 13) Leite e derivados: Leite, iogurte integral e desnatado, iogurte com frutas, requeijão, quei- } \\
\text { jo fresco, muçarela, prato, parmesão }\end{array}$ & 0,382 & 0,439 & 0,071 \\
\hline 14) Sucos: sucos em pó, naturais, artificiais, concentrados, light e diet & 0,086 & 0,359 & 0,044 \\
\hline $\begin{array}{l}\text { 15) Bolachas, bolos e tortas: bolachas doces simples e recheadas, bolos simples e recheados, } \\
\text { tortas doces }\end{array}$ & 0,139 & 0,306 & 0,611 \\
\hline 20) Maionese: maionese, salada de maionese & 0,130 & 0,236 & 0,458 \\
\hline
\end{tabular}

Padrão Tradicional: arroz e massas, óleos, feijões, carnes vermelhas, embutidos e doces. Padrão em Transição: carnes brancas, açúcar, manteiga e margarina, hortaliças e frutas, ovos, pães, leite e derivados, sucos. Padrão Fast Food: bolachas, bolos e tortas, hambúrguer, refrigerantes, chocolate, cafeteria e maionese. 
24 | V.R.M. DISHCHEKENIAN et al.

Tabela 3. Análise de regressão linear múltipla de fatores metabólicos associados a padrões alimentares. São Paulo (SP), 2006.

\begin{tabular}{|c|c|c|c|c|c|c|}
\hline \multirow{2}{*}{ Variáveis } & \multicolumn{2}{|c|}{ Padrão tradicional } & \multicolumn{2}{|c|}{ Padrão em transição } & \multicolumn{2}{|c|}{ Padrão Fast Food } \\
\hline & $\beta$ padr & $p$ & $\beta$ padr & $p$ & $\beta$ padr & $p$ \\
\hline \multicolumn{7}{|l|}{ Glicemia } \\
\hline Sem ajuste & 0,425 & $<0,001$ & 0,258 & 0,014 & 0,066 & 0,523 \\
\hline Sexo/idade/cor & 0,359 & 0,003 & 0,235 & 0,029 & 0,099 & 0,345 \\
\hline Renda/esc mãe/IMC & 0,329 & 0,027 & 0,215 & 0,030 & 0,135 & 0,184 \\
\hline \multicolumn{7}{|l|}{ Insulina } \\
\hline Sem ajuste & 0,304 & $<0,001$ & 0,386 & $<0,001$ & 0,170 & $<0,001$ \\
\hline Sexo /idade/cor & 0,352 & $<0,001$ & 0,417 & $<0,001$ & 0,176 & $<0,001$ \\
\hline Renda/esc mãe/IMC & 0,156 & $<0,001$ & 0,293 & $<0,001$ & 0,028 & 0,226 \\
\hline \multicolumn{7}{|l|}{ Colesterol } \\
\hline Sem ajuste & $-0,065$ & 0,592 & $-0,042$ & 0,727 & 0,243 & 0,001 \\
\hline Sexo/idade/cor & 0,041 & 0,756 & 0,023 & 0,851 & 0,329 & $<0,001$ \\
\hline Renda/esc mãe/IMC & $-0,183$ & 0,169 & $-0,023$ & 0,856 & 0,278 & $<0,001$ \\
\hline \multicolumn{7}{|l|}{ Triglicérides } \\
\hline Sem ajuste & 0,577 & $<0,001$ & 0,414 & $<0,001$ & $-0,126$ & 0,225 \\
\hline Sexo/idade/cor & 0,534 & $<0,001$ & 0,509 & $<0,001$ & $-0,138$ & 0,176 \\
\hline Renda/esc mãe/IMC & 0,513 & $<0,001$ & 0,562 & $<0,001$ & 0,110 & 0,900 \\
\hline \multicolumn{7}{|l|}{$H D L$} \\
\hline Sem ajuste & $-0,270$ & 0,023 & 0,068 & 0,536 & $-0,311$ & $<0,001$ \\
\hline Sexo/idade/cor & $-0,244$ & 0,053 & 0,088 & 0,427 & $-0,350$ & $<0,001$ \\
\hline Renda/esc mãe/IMC & $-0,297$ & 0,020 & 0,136 & 0,234 & $-0,250$ & $<0,001$ \\
\hline \multicolumn{7}{|l|}{$L D L$} \\
\hline Sem ajuste & $-0,093$ & 0,431 & $-0,025$ & 0,835 & 0,314 & $<0,001$ \\
\hline Sexo/idade/cor & 0,001 & 0,991 & 0,170 & 0,887 & 0,354 & $<0,001$ \\
\hline Renda/esc mãe/IMC & $-0,155$ & 0,238 & $-0,510$ & 0,694 & 0,334 & $<0,001$ \\
\hline \multicolumn{7}{|l|}{ PAS } \\
\hline Sem ajuste & 0,173 & 0,090 & $-0,022$ & 0,826 & 0,552 & $<0,001$ \\
\hline Sexo/idade/cor & $-0,175$ & 0,106 & 0,125 & 0,212 & 0,438 & $<0,001$ \\
\hline Renda/esc mãe/IMC & 0,055 & 0,609 & 0,039 & 0,711 & 0,469 & $<0,001$ \\
\hline \multicolumn{7}{|l|}{$P A D$} \\
\hline Sem ajuste & $-0,104$ & 0,130 & 0,198 & 0,033 & 0,504 & $<0,001$ \\
\hline Sexo/idade/cor & $-0,146$ & 0,119 & 0,392 & $<0,001$ & 0,640 & $<0,001$ \\
\hline Renda/esc mãe/IMC & $-0,147$ & 0,129 & 0,328 & 0,001 & 0,615 & $<0,001$ \\
\hline
\end{tabular}

$\beta$ padr: coeficiente de regressão padronizado; Esc mãe: escolaridade da mãe; HDL: lipoproteína de alta densidade; LDL: lipoproteína de baixa densidade; PAS: pressão arterial sistólica; PAD: pressão arterial diastólica; IMC: índice de massa corporal.

escolaridade da mãe e IMC. O padrão "Em transição" apresentou associação significante e positiva com insulina, glicemia, TG e PAD nas três modelagens. O padrão Fast Food apresentou associação significante e positiva com insulina, sem ajuste e com ajuste por sexo, idade e cor. Apresentou ainda associação positiva com o colesterol LDL-C, PAS e PAD nas três modelagens, e negativa com o HDL-C, nas três modelagens.
DIS C U S S Ã O

O padrão Tradicional corresponde aos alimentos presentes nas refeições domiciliares e contempla itens da cesta básica (arroz e outros cereais, tubérculos, leguminosas, carnes e óleo vegetal). Apresenta semelhanças com o padrão tradicional ou convencional relatado em diversos estudos com padrões alimentares ${ }^{11,24,25}$, diferindo 
do padrão tradicional completo, pela ausência de hortaliças e frutas.

O baixo consumo desses alimentos repercute em escassez de vitaminas, minerais e fibras, considerados protetores contra doenças cardiovasculares ${ }^{4}$. Esse comportamento é atribuído ao alto custo, dificuldade de preparo e baixa aceitação por essa população ${ }^{6}$ e pode ser confirmado em trabalho que apontou maior consumo de doces e guloseimas, bem como pequeno consumo de hortaliças e frutas, entre crianças e jovens de baixo nível socioeconômico e baixa escolaridade materna ${ }^{18}$.

Além dos produtos tradicionais, o presente estudo aponta a provável substituição da carne por produtos como a salsicha e a linguiça, alimentos que tiveram seu consumo aumentado na população, de acordo com a Pesquisa de Orçamentos Familiares ${ }^{7}$, muitas vezes pela praticidade e baixo custo.

A associação positiva do padrão Tradicional com glicemia, insulina e triglicérides pode ser justificada pelo consumo de arroz, macarrão, batata, doces e alimentos refinados, ricos em carboidratos de absorção rápida ${ }^{26}$, acessíveis e consumidos em grandes quantidades. A associação negativa desse padrão com o HDLc pode ser explicada pelas repercussões metabólicas desencadeadas por esse tipo de dieta.

Mikkila et al. ${ }^{5}$ encontraram em seu estudo finlandês Cardiovascular Risk in Young Finns Study associação positiva de um padrão similar ao Tradicional, com fatores de risco cardiovascular. Outro estudo mostrou ainda a associação de uma dieta rica em carboidratos simples, de absorção rápida e pobre em fibras oriundas dos cereais e frutas, com risco aumentado para diabetes tipo $2^{27}$.

O padrão Em Transição apresentou associações positivas com glicemia, insulina, TG e PAD. Embora se trate de um grupo de adolescentes obesos reconhecidamente com erros alimentares, esse padrão parece agregar indivíduos que estavam fazendo refeições supostamente saudáveis e substituição das grandes refeições por lanches preparados em casa ou em lanchonetes, na tentativa de melhorar a saúde e emagrecer.

Ele poderia ser considerado um padrão com composição saudável devido a alguns componentes, porém as associações encontradas apontam que a presença desses alimentos não foi suficiente para evitar alterações no metabolismo dos carboidratos. Muitas vezes o adolescente apresenta um hábito alimentar inconsistente, omitindo refeições ou substituindo-as por lanches; provavelmente na tentativa de diminuir o consumo energético ${ }^{6}$. Esse padrão contempla alimentos saudáveis como carnes magras, legumes, verduras e frutas, associados ao consumo de pães, manteiga, suco de fruta natural e artificial e açúcar refinado, sendo este último normalmente adicionado a leite e sucos. Corroborando esses achados, estudos europeus e americanos apontam que o consumo do açúcar de adição vem crescendo, variando de 14 a 29\%, e sendo inversamente associado ao consumo de gorduras, vitaminas e minerais ${ }^{28}$. Padrão alimentar com composição similar foi encontrado em estudos com adolescentes mexicanas ${ }^{29}$, crianças coreanas ${ }^{25}$ e francesas ${ }^{30}$.

A associação da PAD com o padrão Em transição poderia ser parcialmente atribuída a altos teores de sal presentes na manteiga e margarina, sucos em pó e sal de adição a verduras e legumes ${ }^{14}$. Tratando-se de um grupo de adolescentes obesos, os grandes volumes consumidos poderiam ser a justificativa dessa alteração clínica.

O padrão Fast Food apresentou associações positivas com o colesterol total e LDL-C, e negativa com o HDL-c. Isso pode ser confirmado por outros estudos e justificado pela presença de altos níveis de gordura saturada e trans, assim como de baixos teores de fibras nos alimentos desse grupo ${ }^{16,24}$. Esse padrão apresentou ainda associação positiva com a insulina, bem como os demais padrões, provavelmente porque, entre os obesos em geral, a primeira alteração metabólica associada à resistência insulínica é a hiperinsulinemia. Não houve associação desse padrão com a glicemia, supostamente por sua alteração ser 
uma manifestação mais tardia para a faixa etária em estudo. Acredita-se ainda que o consumo de alimentos com alto teor de gorduras associado a carboidratos simples tenha menor influência sobre os níveis de glicemia do que nos demais padrões, uma vez que tal consumo parece retardar o tempo de absorção da glicose, resultando numa preservação dos níveis glicêmicos ${ }^{13}$.

A associação positiva do padrão Fast Food com a PAS e PAD poderia ser justificada pelos altos níveis de sódio existentes nos produtos de cafeteria (salgados e lanches prontos), alimentos industrializados (salgadinhos, amendoim, molhos prontos) ou sal de adição (pipoca, batata-frita). Dietas pobres em hortaliças, frutas e produtos lácteos, e ricas em gordura e sal, parecem estar associadas a essa alteração ${ }^{26}$. Entre os fatores ambientais, a dieta parece exercer o principal papel no desenvolvimento da hipertensão arterial4,15.

Esse padrão pode ser associado à alimentação fora de casa, na escola (cantina e "carrocinhas") e nos momentos de lazer (lanchonetes, quiosques, restaurantes), assim como à alta palatabilidade, aceitação, praticidade e status, características essenciais na escolha alimentar do adolescente. Além disso, os lanches vêm crescendo em nível doméstico, sendo comumente apontados pelas famílias como substitutos da refeição notur$n a^{6}$. Acredita-se que uma limitação para o consumo em maior escala desse padrão, pela população em estudo, seja o seu alto custo.

Avaliando-se o consumo alimentar geral da população, nos últimos anos vem sendo recomendada uma diminuição do consumo da gordura dietética saturada, com o objetivo de prevenir doenças cardiovasculares, resultando em dietas hipolipídicas e automaticamente hiperglicídicas, com mais de $60 \%$ do Valor Energético Total (VET) na forma de carboidratos. A compensação normalmente tem sido feita pelo consumo de carboidratos simples, geralmente processados (arroz refinado, batata, macarrão, pães), em vez dos carboidratos complexos preconizados, encontrados em grãos e farinhas integrais, hortaliças e frutas.
Enquanto os efeitos deletérios das dietas hiperlipídicas apresentam-se bem estabelecidos, porém transmitidos à população de maneira ineficaz, dietas ricas em carboidratos vêm sendo promovidas mundialmente por órgãos oficiais de saúde. O Guia Alimentar para a População Brasileira preconiza o consumo de $55 \%$ a $75 \%$ do VET na forma de carboidratos ${ }^{31}$.

Estudos vêm apontando que a substituição de gorduras por carboidratos desenvolve uma alteração metabólica ainda pior se eles forem refinados, especialmente em indivíduos sedentários, com sobrepeso, resistência insulínica e predisposição familiar ${ }^{4}$, associando-se com hipertrigliceridemia, aumento da lipogênese e da formação das LDLC pequenas e densas, com consequente aumento da adiposidade e redução nos níveis de HDLc plasmático ${ }^{24,32}$.

A fisiologia humana não foi estruturada para suportar as alterações hormonais expressas por rápidas elevações da glicemia e insulina, causadas pelo consumo de alimentos modernos, ricos em carboidratos simples, gorduras saturadas e trans, cada vez mais presentes na dieta. Quanto maior e mais rápida a alteração do steady state presente nas situações de jejum, maiores as alterações e os efeitos produzidos. Provavelmente a demanda aumentada de insulina de maneira crônica resulte numa exaustão pancreática que pode desencadear uma intolerância à glicose ${ }^{27}$. Sendo assim, todos os efeitos fisiológicos do carboidrato dietético, a longo prazo, poderiam ser minimizados se a sua digestão e absorção fossem mais lentas ${ }^{13,26}$.

Nas últimas décadas muito se descobriu sobre os efeitos da dieta na saúde do indivíduo, porém, apesar de os comportamentos considerados saudáveis serem conhecidos, a prevalência das doenças crônicas, em especial a obesidade e o diabetes, só vem aumentando na maioria dos países desenvolvidos e em desenvolvimento. Provavelmente grande parte da população mundial enfrentará as doenças crônicas, devido ao aumento da expectativa de vida conquistado ${ }^{4}$. 
Entre as diversas limitações deste estudo, cabe apontar a falta de trabalhos acerca de padrões alimentares de crianças e adolescentes, levando a comparações com dados de adultos, em muitas situações.

Os padrões alimentares parecem variar com o sexo, idade, cultura, etnia e condição socioeconômica, o que faz necessário analisá-los em diferentes populações, para verificar sua aplicabilidade. Além disso, mudanças na preferência alimentar e disponibilidade de alimentos fazem com que a interpretação dos padrões alimentares possa variar com o tempo ${ }^{12}$.

Considerando-se a homogeneidade da população estudada no que se refere a idade, condição socioeconômica e estado nutricional, sugere-se que os resultados sejam extrapolados exclusivamente para populações com características semelhantes.

Além disso, imprecisão na quantificação do consumo pode ser atribuída ao sub-relato ou à redução do consumo nos dias de coleta de dados. Tal limitação é citada em vários estudos e pode ser apontada como frequente em indivíduos com excesso de peso, que desejam perder peso ou que passaram por regimes de restrição alimentar. Estudos apontam para a existência de sub-relato seletivo de consumo alimentar para alimentos ricos em gordura e colesterol, possivelmente devido aos frequentes alertas para os efeitos deletérios de seu consumo para a saúde ${ }^{33}$.

Pesquisa utilizando biomarcadores para quantificar o sub-relato de consumo alimentar em diversos instrumentos de levantamento dietético, apontou $13 \%$ de sub-relato em recordatórios de 24 horas múltiplos e 33\% em questionários de frequência ${ }^{34}$.

Apesar das limitações dos métodos de investigação da dieta habitual, em razão da pouca praticidade, falta de validação, baixa abrangência das tabelas de composição nutricional, assim como diversidade na composição dos produtos industrializados, regionalismo dos hábitos alimentares, dificuldade de identificação e quantificação dos alimentos pelo paciente e possibilidade de sub-relato, sua utilização é de fundamental importância em epidemiologia nutricional 19,34.

No presente estudo, as escolhas e combinações alimentares variavam muito entre os adolescentes, o que foi de fundamental importância na decisão de estudar os padrões alimentares dessa população.

\section{O N CLUS Ã O}

Os padrões alimentares observados nesse grupo de adolescentes foram considerados inadequados.

As alterações bioquímicas e dos níveis pressóricos, encontradas na amostra, provavelmente se devem aos excessos alimentares verificados. Os padrões Tradicional e Em transição associaram-se à hiperglicemia, hiperinsulinemia e hipertrigliceridemia, enquanto o padrão Fast Food associou-se às alterações lipídicas, hiperinsulinemia e hipertensão arterial. Os três padrões podem ser considerados obesogênicos, parecendo ser o padrão Fast Food o mais aterogênico e promotor de hipertensão arterial.

\section{CONSIDERAÇÕES FINAIS}

Cada vez mais parece claro que não é a presença ou ausência de um determinado alimento que se associa com a saúde ou a doença, mas sim o conjunto de alimentos consumidos, levando-se em conta a escolha criteriosa, a quantidade e as proporções adequadas.

Identificar os alimentos mais aceitos, depois agrupá-los em padrões por frequência e volume de consumo, e examinar as associações entre eles e as alterações metabólicas associadas às doenças crônicas, ajuda na identificação de áreas de intervenção e no planejamento de estratégias de mudança de comportamento alimentar individual e coletivo.

Mudanças comportamentais pressupõem ruptura de hábitos familiares com fortes raízes 
socioculturais e, portanto, requerem políticas de prevenção e promoção de saúde que valorizem e estimulem a alimentação saudável e combatam os padrões alimentares nocivos apontados, uma vez que hábitos alimentares incorporados na infância e acentuados na adolescência propiciam o aparecimento ou agravo de fatores biológicos passíveis de reversão.

Mais ênfase deve ser dada às mudanças qualitativas, como a redução do consumo de gorduras saturadas e trans, e o aumento do consumo de gorduras poli e monoinsaturadas (óleos vegetais, sementes e oleaginosas). Sugere-se ainda uma revisão da distribuição dos macronutrientes dentro do valor energético diário e propostas de substituição de carboidratos simples, gorduras saturadas e trans, uma vez que o consumo apontado no estudo estava dentro dos níveis preconizados pelos guias alimentares nacionais, porém associado com alterações metabólicas.

\section{OLABORADORES}

V.R.M. DISHCHEKENIAN responsável pela idealização do trabalho, coleta e análise de dados e redação do trabalho. M.A.M.S ESCRIVÃO coordenadora do projeto, responsável pela idealização do trabalho, discussão e interpretação de dados. D. PALMA corresponsável pela idealização do trabalho. F. ANCONA-LOPEZ corresponsável pela idealização do trabalho. E.A.C. ARAÚJO responsável pela análise estatística e discussão dos resultados. J.A.A.C. TADDEl responsável pela idealização do trabalho, revisão e discussão das análises de dados e redação final.

\section{REFERÊNCIAS}

1. World Health Organization. Adolescent nutrition: a neglected dimension. 2003 [cited 2008 Dec 3]. Available from: <http://www.who.int/nut/ado. htm.2>.

2. Schwerin HS, Stanton JL, Riley Jr AM, Schaefer AE, Leveille GA, Elliot JG, et al. Food eating patterns and health: a reexamination of the Ten-States and HANES I surveys. Am J Clin Nutr. 1981; 34(4): 568-80.
3. McNaughton SA, Ball K, Mishra GD, Crawford DA. Dietary patterns of adolescents and risk of obesity and hypertension. J Nutr. 2008; 138(2):364-70.

4. Willet WC. Overview and perspective in human nutrition. Asia Pac J Clin Nutr. 2008; 17(S1):1-4.

5. Mikkila V, Rasanen L, Raitakari OT, Marniemi J, Pietinen P, Ronnemaa T, et al. Major dietary patterns and cardiovascular risk factors from childhood to adulthood. The Cardiovascular Risk in Young Finns Study. Br J Nutr. 2007; 98(1):218-25. doi:10.1017/ S0007114507691831.

6. Nicklas TA, Baranowski T, Cullen KW, Berenson G. Eating patterns, dietary quality and obesity. J Am Coll Nutr. 2001; 20(6):599-608.

7. Instituto Brasileiro de Geografia e Estatística. Pesquisa de orçamentos familiares 2002-2003: antropometria e análise do estado nutricional de crianças e adolescentes no Brasil. Rio de Janeiro: IBGE; 2006.

8. Escrivão MAMS, Oliveira FLC, Taddei JAAC, Ancona-Lopez F. obesidade exógena na infância e adolescência. J Ped. 2000; 76(3):305-10.

9. Kac G, Sichieri R, Gigante DP. Epidemiologia nutricional. Rio de Janeiro: Fiocruz; 2007.

10. Willet WC, Howe GR, Kushi LH. Adjustments for total energy intake in epidemiologic studies. Am J Cin Nutr. 1997; 65(4):1220S-8S.

11. Andrade RG, Pereira RA, Sichieri R. Consumo alimentar de adolescentes com e sem sobrepeso do Município do Rio de Janeiro. Cad Saúde Pública. 2003; 19(5):1485-95. doi: 10.1590/S0102-311X2 003000500027.

12. Hu FB. Dietary pattern analysis: a new direction in nutritional epidemiology. Curr Opin Lipidol. 2002; 13(1):3-9.

13. Polacow V, Lancha Jr AH. Dietas hiperglicídicas: efeitos da substituição isoenergética de gordura por carboidratos sobre o metabolismo de lipídeos, adiposidade corporal e sua associação com atividade física e com o risco de doença cardiovascular. Arq Bras Endocrinol Metab. 2007; 51(3):389-400. doi: 10.1590/S0004-27302007000300006.

14. Ricardi G, Rivellese AA. Dietary treatment of the metabolic syndrome- the optimal diet. Br J Nutri. 2000; 83(Suppl 1):S143-8. doi:10.1017/S000711 4500001082.

15. Slattery ML. Defining dietary consumption: is the sum greater than its parts? Am J Clin Nutr. 2008; 88(1):14-5.

16. Song $Y$, Joung $H$, Engelhardt $K$, Sun $Y Y$, Paik $H Y$. Traditional vs modified dietary patterns and their influence on adolescents nutritional profile. $\mathrm{Br} \mathrm{J}$ Nutr. 2005; 93(6):943-49. doi:10.1079/BJN20051 435. 
17. Ritchie LD, Spector $P$, Stevens MJ, Schmidt MM, Schreiber GB, Striegel-Moore RH, et al. Dietary patterns in adolescence are related to adiposity in young adulthood in black and white females. J Nutr. 2007; 137(2):399-406.

18. Aranceta J, Pérez-Rodrigo C, Ribas L, Serra-Majem L. Sociodemographic and lifestyle determinants of food patterns in Spanish children and adolescents: the enKid study. Eur J Clin Nutr. 2003; 57 (Suppl 1):S40-4. doi: 10.1038/SJ.EJCN.1601813.

19. Must A, Dallal GE, Dietz WH. Reference data for obesity: 85th and 95th percentiles of body mass index (wt/ht2) and triceps skinfold thickness. Am J Clin Nutr. 1991; 53(4):839-846 [published erratum appears in Am J Clin Nutr. 1991; 54(5): 773].

20. Tanner JM. Growth at adolescence. $2^{\text {nd }}$ ed. Oxford, UK: Blackwell Scientific Publications; 1962.

21. National Research Council. Food and Nutrition Board. Recommended Dietary Allowances. $10^{\text {th }}$ ed. Washington (DC): National Academy Press; 1989.

22. Hair JF, Anderson RE, Tatham RL, Black WC. Multivariate data analysis. $5^{\text {th }}$ ed. New Jersey: Prentice Hall; 1998.

23. O'Connor BP. SPSS and SAS programs for determining the number of components using parallel analysis and Velicer's MAP test. Behav Res Methods. 2000; 32:396-402.

24. Neumann AICP, Martins IS, Marcopito LF, Araujo EAC. Padrões alimentares associados a fatores de risco para doenças cardiovasculares entre residentes de um município brasileiro. Rev Panam Salud Publica. 2007; 22(5):329-39. doi: 10.1590/S1020-49 892007001000006.

25. Li SJ, Paik HY, Joung $H$. Dietary patterns are associated with sexual maturation in Korean children. Br J Nutr. 2006; 95(4):817-23. doi: 10.1079/BJN20051675.

26. Steemburgo T, Dall'Alba V, Gross JL, Azevedo MJ. Fatores dietéticos e síndrome metabólica. Arq Bras Endocrinol Metab. 2007; 51(9):1425-33. doi: 10.15 90/S0004-27302007000900004.
27. Schulze MB, Liu S, Rimm EB, Manson JA, Willet WD, Hu FB. Glycemic index, glycemic load and dietary fiber intake and incidence of 2 diabetes in younger and middle-aged women. Am J Clin Nutr. 2004; 80(2):348-56.

28. Alexy U, Sichert-Hellert W, Kersting M. Associations between intake of added sugars and intakes of nutrients and food groups in the diets of German children and adolescents. Br J Nutr. 2003; 90(2): 441-7. doi:10.1079/BJN2003904.

29. Lozada AL, Flores M, Rodriguez S, Barquera S. [Dietary patterns in Mexican adolescent girls. A comparison of two methods. National Nutrition Survey, 1999]. Salud Publica Mex. 2007; 49(4): 263-73. doi: 10.1590/50036-363420070004000 06.

30. Lioret S, Touvier M, Lafay L, Volatier JL, Maire B. Dietary and physical activity patterns in French children are related to overweight and socioeconomic status. J Nutr. 2008; 138(1):101-7.

31. Brasil. Ministério da Saúde. Secretaria de Atenção à Saúde. Guia alimentar para a população brasileira: promovendo a alimentação saudável. Brasília: Ministério da Saúde; 2005.

32. Risérus $U$, Willet WD, Hu FB. Dietary fats and prevention of type 2 diabetes. Prog Lipid Res. 2009; 48(1):44-51. doi:10.1016/j. plipres.2008.10.002.

33. Goris AH, Westerterp-Plantenga MS, Westerterp KR. Undereating and underrecording of habitual food intake in obese men: selective underreporting of fat intake. Am J Clin Nutr. 2000; 71(1):130-4.

34. Subar AF, Kipnis V, Troiano RP, Midthune D, Schoeller DA, Bingham $S$, et al. Using intake biomarkers to evaluate the extent of dietary misreporting in a large sample of adults: the OPEN study. Am J Epidemiol. 2003; 158(1):1-13. doi: 10.1093/aje/kwg092.

Recebido em: 16/2/2009

Versão final reapresentada em: 24/2/2010 Aprovado em: 31/5/2010 
